\title{
Association between ABCC8 Ala1369Ser Polymorphism (rs757110 T/G) and Type 2 Diabetes Risk in an Iranian Population: A Case-Control Study
}

\author{
Amin Bakhtiyari ${ }^{1}$, Karimeh Haghani ${ }^{2}$, Salar Bakhtiyari ${ }^{2 *}$, Mohammad A. Zaimy ${ }^{3}$, Nooriali Zahed ${ }^{4}$, Ali \\ Gheysarzadeh $^{5}$, Shahram Darabi ${ }^{6}$, Seidali Nahalkhani², Mansour Amraei ${ }^{7}$, Iraj Alipourfard ${ }^{8}$ \\ ${ }^{1}$ Department of Genetics, Biology Research Center, Zanjan Branch, Islamic Azad University, Zanjan, Iran. \\ ${ }^{2}$ Department of Clinical Biochemistry, Faculty of Medicine, Ilam University of Medical Sciences, Ilam, Iran. \\ ${ }^{3}$ Department of Medical Genetics, Faculty of Medicine, Ilam University of Medical Sciences, Ilam, Iran. \\ ${ }^{4}$ Department of Clinical Biochemistry, Faculty of Allied Medical Sciences, Ilam University of Medical \\ Sciences, Ilam, Iran. \\ ${ }^{5}$ Clinical Microbiology Research Center, Ilam University of Medical Sciences, Ilam, Iran. \\ ${ }^{6}$ Cellular and Molecular Research Center, Qazvin University of Medical Sciences, Qazvin, Iran. \\ ${ }^{7}$ Department of Physiology, Faculty of Medicine, Ilam University of Medical Sciences, Ilam, Iran. \\ ${ }^{8}$ School of Pharmacy, Faculty of Sciences, University of Rome Tor Vergata, Rome, Italy. \\ DOI: $10.2174 / 1871530320666200713091827$
}

\begin{abstract}
Objective: Glucose metabolism increases ATP/ADP ratio within the $\beta$-cells and causes ATP-sensitive K+ (KATP) channel closure and consequently insulin secretion. The enhanced activity of the channel may be a mechanism contributing to the reduced first-phase of insulin secretion observed in T2DM. There is no study to date in the Kurdish ethnic group regarding the relationship between SNP Ala1369Ser (rs757110 T/G) of SUR1 gene and T2DM, and additionally, the results of this association in other populations are inconsistent. Therefore, our aim in this study was to explore the possible association between SNP Ala1369Ser and type 2 diabetes in an Iranian Kurdish ethnic group.

Methods: In this study, we checked out the frequency of alleles and genotypes of SNP Ala1369Ser in T2DM individuals (207 patients; men/women: 106/101) and non-T2DM subjects (201 controls; men/women: 97/104), and their effects on anthropometric, clinical, and biochemical parameters. Genomic DNA was extracted from the leukocytes of blood specimens using a standard method. We amplified the ABCC8 rs757110 polymorphic site $(\mathrm{T} / \mathrm{G})$ using a polymerase chain reaction (PCR) method and a designed primer pair. To perform the PCR-RFLP method, the amplicons were subjected to restriction enzymes and the resulting fragments separated by gel electrophoresis.

Results: The frequency of the G-allele of Ala1369Ser polymorphism was significantly (0.01) higher in the case group than the control group (19\% vs. $9 \%$, respectively). In the dominant model (TT vs. TG+GG), there was a significant relationship between this SNP and an increased risk of T2DM $(\mathrm{P}=0.00)$. T2DM patients with TG+GG genotypes had significantly higher fasting plasma insulin and HOMA-IR than those who had the TT genotype (P $=0.02$ and 0.01 , respectively).

Conclusion: Our study is the first study to investigate the association between Ala1369Ser ABCC8 genetic variation and T2DM in the Kurdish population of western Iran. The obtained results clearly show that Ala1369Ser polymorphism of ABCC8 is associated with an increased risk of T2DM in this population.
\end{abstract}

Keywords: ABCC8; KATP channel; SNP rs757110; SUR1; T2DM; diabetes. 